\title{
ON THE STRUCTURE TENSORS OF ALMOST CONTACT B-METRIC MANIFOLDS
}

\author{
HRISTO MANEV
}

\begin{abstract}
Aвstract. The space of the structure (0,3)-tensors of the covariant derivatives of the structure endomorphism and the metric on almost contact B-metric manifolds is considered. A known decomposition of this space in orthogonal and invariant subspaces with respect to the action of the structure group is used. We determine the corresponding components of the structure tensor and consider the case of the lowest dimension 3 of the studied manifolds. Some examples are commented.
\end{abstract}

\section{INTRODUCTION}

The idea of decomposition of the space of the structure $(0,3)$-tensors, generated by the covariant derivative of the fundamental tensor of type $(1,1)$, is used by different authors in order to obtain classifications of manifolds with additional tensor structures. For example, let us mention the classification of almost Hermitian manifolds given in [4], of almost complex manifolds with Norden metric - in [2], of almost contact metric manifolds - in [1], of almost contact manifolds with B-metric - in [3], of Riemannian almost product manifolds - in [8], of Riemannian manifolds with traceless almost product structure - in [10], of almost paracontact metric manifolds - in [7], of almost paracontact Riemannian manifolds of type $(n, n)-$ in [6].

The almost contact structure is well studied, especially in the case when it is equipped with a compatible Riemannian (or pseudo-Riemannian) metric, i.e. the almost contact endomorphism $\varphi$ acts as an isometry with respect to the metric in each tangent fibre of the contact distribution $H=\operatorname{ker}(\eta)$, where $\eta$ is the contact 1-form. An indefinite counterpart is the almost contact B-metric structure, i.e. $\varphi$ acts as an anti-isometry with respect to B-metric in $H$.

The goal of this work is the description of the structure tensor generated by the covariant derivative of $\varphi$ and the B-metric by its components in the different basic classes of the classification of the almost contact B-metric manifolds made by G. Ganchev, V. Mihova, K. Gribachev in [3]. The case of the lowest dimension 3 of the studied manifolds is considered and it is establish that four of the basic classes are restricted to the spacial class with zero structure tensors.

The paper is organized as follows. In Sect. 1 we recall some facts about the almost contact manifolds with B-metric. In Sect. 2 we decompose the vector space of the structure tensors on the considered manifolds. In Sect. 3 we deduce the components of the structure tensor in the case of the lowest dimension 3. In Sect. 4 we comment some examples in relation with the above investigations.

\section{Almost contact manifolds with B-metric}

Let $(M, \varphi, \xi, \eta, g)$ be an almost contact manifold with B-metric or an almost contact B-metric manifold, i.e. $M$ is a $(2 n+1)$-dimensional differentiable manifold with an almost contact structure $(\varphi, \xi, \eta)$ consisting of an endomorphism $\varphi$ of the tangent bundle, a vector field $\xi$, its dual 1-form $\eta$ as well as $M$ is equipped with a pseudo-Riemannian metric $g$ of signature $(n+1, n)$, such that the following algebraic relations are satisfied: [3]

$$
\varphi \xi=0, \quad \varphi^{2}=-\mathrm{Id}+\eta \otimes \xi, \quad \eta \circ \varphi=0, \quad \eta(\xi)=1, \quad g(\varphi x, \varphi y)=-g(x, y)+\eta(x) \eta(y)
$$

2010 Mathematics Subject Classification. Primary 53C15, 53C50; Secondary 53D15.

Key words and phrases. almost contact manifold, B-metric, structure tensor.

This paper is partially supported by project NI13-FMI-002 of the Scientific Research Fund, Plovdiv University, Bulgaria 
for any $x, y$ of the algebra $\mathfrak{X}(M)$ of the smooth vector fields on $M$. Further $x, y, z$ will stand for arbitrary elements of $\mathfrak{X}(M)$ or vectors in the tangent space $T_{p} M$ at $p \in M$.

The associated metric $\tilde{g}$ of $g$ on $M$ is defined by $\tilde{g}(x, y)=g(x, \varphi y)+\eta(x) \eta(y)$. The manifold $(M, \varphi, \xi, \eta, \tilde{g})$ is also an almost contact B-metric manifold. Both metrics $g$ and $\tilde{g}$ are necessarily of signature $(n+1, n)$. The Levi-Civita connection of $g$ and $\tilde{g}$ will be denoted by $\nabla$ and $\tilde{\nabla}$, respectively.

Let us denote the structure group of $(M, \varphi, \xi, \eta, g)$ by $\mathcal{G}$. It is determined by $\mathcal{G}=O(n ; \mathbb{C}) \times \mathcal{I}$, where $\mathcal{I}$ is the identity on $\operatorname{span}(\xi)$ and $O(n ; \mathbb{C})=\mathcal{G} \mathcal{L}(n ; \mathbb{C}) \cap O(n, n)$, i.e. $\mathcal{G}$ consists of the real square matrices of order $2 n+1$ of the following type

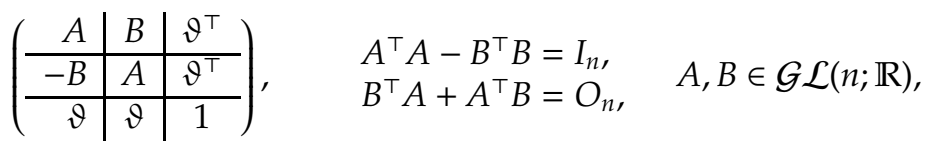

where $\vartheta$ and its transpose $\vartheta^{\top}$ are the zero row $n$-vector and the zero column $n$-vector; $I_{n}$ and $O_{n}$ are the unit matrix and the zero matrix of size $n$, respectively.

A classification of the almost contact B-metric manifolds is given in [3]. This classification, consisting of eleven basic classes $\mathcal{F}_{1}, \mathcal{F}_{2}, \ldots, \mathcal{F}_{11}$, is made with respect to the tensor $F$ of type $(0,3)$ defined by

$$
F(x, y, z)=g\left(\left(\nabla_{x} \varphi\right) y, z\right)
$$

which have the following properties

$$
F(x, y, z)=F(x, z, y)=F(x, \varphi y, \varphi z)+\eta(y) F(x, \xi, z)+\eta(z) F(x, y, \xi) .
$$

The intersection of the basic classes is the special class $\mathcal{F}_{0}$ determined by the condition $F(x, y, z)=$ 0 . Hence $\mathcal{F}_{0}$ is the class of the almost contact B-metric manifolds with $\nabla$-parallel structures, i.e. $\nabla \varphi=\nabla \xi=\nabla \eta=\nabla g=\nabla \tilde{g}=0$.

If $\left\{e_{i} ; \xi\right\}(i=1,2, \ldots, 2 n)$ is a basis of $T_{p} M$ and $\left(g^{i j}\right)$ is the inverse matrix of the matrix $\left(g_{i j}\right)$ of $g$, then the following 1 -forms are associated with $F$ :

$$
\theta(z)=g^{i j} F\left(e_{i}, e_{j}, z\right), \quad \theta^{*}(z)=g^{i j} F\left(e_{i}, \varphi e_{j}, z\right), \quad \omega(z)=F(\xi, \xi, z) .
$$

These 1-forms are known also as the Lee forms. Obviously, the identities $\omega(\xi)=0$ and $\theta^{*} \circ \varphi=$ $-\theta \circ \varphi^{2}$ are always valid.

Further we use the following characteristic conditions of the basic classes: [5]

$$
\begin{array}{ll}
\mathcal{F}_{1}: & F(x, y, z)=\frac{1}{2 n}\left\{g(x, \varphi y) \theta(\varphi z)+g(\varphi x, \varphi y) \theta\left(\varphi^{2} z\right)+g(x, \varphi z) \theta(\varphi y)+g(\varphi x, \varphi z) \theta\left(\varphi^{2} y\right)\right\} \\
\mathcal{F}_{2}: & F(\xi, y, z)=F(x, \xi, z)=0, \quad F(x, y, \varphi z)+F(y, z, \varphi x)+F(z, x, \varphi y)=0, \quad \theta=0 ; \\
\mathcal{F}_{3}: & F(\xi, y, z)=F(x, \xi, z)=0, \quad F(x, y, z)+F(y, z, x)+F(z, x, y)=0 ; \\
\mathcal{F}_{4}: & F(x, y, z)=-\frac{1}{2 n} \theta(\xi)\{g(\varphi x, \varphi y) \eta(z)+g(\varphi x, \varphi z) \eta(y)\} ; \\
\mathcal{F}_{5}: & F(x, y, z)=-\frac{1}{2 n} \theta^{*}(\xi)\{g(x, \varphi y) \eta(z)+g(x, \varphi z) \eta(y)\} ; \\
\mathcal{F}_{6}: & F(x, y, z)=F(x, y, \xi) \eta(z)+F(x, z, \xi) \eta(y), \\
& F(x, y, \xi)=F(y, x, \xi)=-F(\varphi x, \varphi y, \xi), \quad \theta=\theta^{*}=0 ; \\
\mathcal{F}_{7}: & F(x, y, z)=F(x, y, \xi) \eta(z)+F(x, z, \xi) \eta(y), \quad F(x, y, \xi)=-F(y, x, \xi)=-F(\varphi x, \varphi y, \xi) \\
\mathcal{F}_{8}: & F(x, y, z)=F(x, y, \xi) \eta(z)+F(x, z, \xi) \eta(y), \quad F(x, y, \xi)=F(y, x, \xi)=F(\varphi x, \varphi y, \xi) ; \\
\mathcal{F}_{9}: & F(x, y, z)=F(x, y, \xi) \eta(z)+F(x, z, \xi) \eta(y), \quad F(x, y, \xi)=-F(y, x, \xi)=F(\varphi x, \varphi y, \xi) ; \\
\mathcal{F}_{10}: & F(x, y, z)=F(\xi, \varphi y, \varphi z) \eta(x) ; \\
\mathcal{F}_{11}: & F(x, y, z)=\eta(x)\{\eta(y) \omega(z)+\eta(z) \omega(y)\} .
\end{array}
$$




\section{A DECOMPOSITION OF THE SPACE OF THE STRUCTURE TENSORS}

Let us consider $T_{p} M$ at arbitrary $p \in M$ as a $(2 n+1)$-dimensional vector space equipped with almost contact B-metric structure $(\varphi, \xi, \eta, g)$. Let $\mathcal{F}$ be the vector space of all tensors $F$ of type $(0,3)$ over $T_{p} M$ having properties (3), i.e.

(6) $\mathcal{F}=\{F(x, y, z) \in \mathbb{R} \mid F(x, y, z)=F(x, z, y)=F(x, \varphi y, \varphi z)+\eta(y) F(x, \xi, z)+\eta(z) F(x, y, \xi)\}$.

The metric $g$ induces an inner product $\langle\cdot, \cdot\rangle$ on $\mathcal{F}$ defined by

$$
\left\langle F^{\prime}, F^{\prime \prime}\right\rangle=g^{i q} g^{j r} g^{k s} F^{\prime}\left(e_{i}, e_{j}, e_{k}\right) F^{\prime \prime}\left(e_{q}, e_{r}, e_{s}\right)
$$

for any $F^{\prime}, F^{\prime \prime} \in \mathcal{F}$ and a basis $\left\{e_{i}\right\}(i=1,2, \ldots, 2 n+1)$ of $T_{p} M$.

The standard representation of the structure group $\mathcal{G}$ in $T_{p} M$ induces a natural representation $\lambda$ of $\mathcal{G}$ in $\mathcal{F}$ as follows

$$
((\lambda a) F)(x, y, z)=F\left(a^{-1} x, a^{-1} y, a^{-1} z\right)
$$

for any $a \in \mathcal{G}$ and $F \in \mathcal{F}$, so that for $F^{\prime}, F^{\prime \prime} \in \mathcal{F}$

$$
\left\langle(\lambda a) F^{\prime},(\lambda a) F^{\prime \prime}\right\rangle=\left\langle F^{\prime}, F^{\prime \prime}\right\rangle \text {. }
$$

The decomposition $x=-\varphi^{2} x+\eta(x) \xi$ generates the projectors $h$ and $v$ on $T_{p} M$ determined by $h(x)=-\varphi^{2} x$ and $v(x)=\eta(x) \xi$, having the properties $h \circ h=h, v \circ v=v, h \circ v=v \circ h=0$.

Therefore, we have the orthogonal decomposition $T_{p} M=h\left(T_{p} M\right) \oplus v\left(T_{p} M\right)$.

Bearing in mind these projectors on $T_{p} M$, we construct a partial decomposition of $\mathcal{F}$ as follows.

At first, we define the operator $p_{1}: \mathcal{F} \rightarrow \mathcal{F}$ by

$$
p_{1}(F)(x, y, z)=-F\left(\varphi^{2} x, \varphi^{2} y, \varphi^{2} z\right)
$$

It is easy to check the following

Lemma 2.1. The operator $p_{1}$ has the following properties:

(i) $p_{1} \circ p_{1}=p_{1}$;

(ii) $\left\langle p_{1}\left(F^{\prime}\right), F^{\prime \prime}\right\rangle=\left\langle F^{\prime}, p_{1}\left(F^{\prime \prime}\right)\right\rangle, \quad F^{\prime}, F^{\prime \prime} \in \mathcal{F}$;

(iii) $p_{1} \circ(\lambda a)=(\lambda a) \circ p_{1}$.

According to Lemma 2.1 we have the following orthogonal decomposition of $\mathcal{F}$ by the image and the kernel of $p_{1}$ :

$$
\mathcal{W}_{1}=\operatorname{im}\left(p_{1}\right)=\left\{F \in \mathcal{F} \mid p_{1}(F)=F\right\}, \quad \mathcal{W}_{1}^{\perp}=\operatorname{ker}\left(p_{1}\right)=\left\{F \in \mathcal{F} \mid p_{1}(F)=0\right\} .
$$

Further, we consider the operator $p_{2}: \mathcal{W}_{1}^{\perp} \rightarrow \mathcal{W}_{1}^{\perp}$ defined by

$$
p_{2}(F)(x, y, z)=\eta(y) F\left(\varphi^{2} x, \xi, \varphi^{2} z\right)+\eta(z) F\left(\varphi^{2} x, \varphi^{2} y, \xi\right)
$$

for which we obtain

Lemma 2.2. The operator $p_{2}$ has the following properties:

(i) $p_{2} \circ p_{2}=p_{2}$;

(ii) $\left\langle p_{2}\left(F^{\prime}\right), F^{\prime \prime}\right\rangle=\left\langle F^{\prime}, p_{2}\left(F^{\prime \prime}\right)\right\rangle, \quad F^{\prime}, F^{\prime \prime} \in \mathcal{W}_{1}^{\perp}$;

(iii) $p_{2} \circ(\lambda a)=(\lambda a) \circ p_{2}$.

Then, bearing in mind Lemma2.2, we obtain

$$
\mathcal{W}_{2}=\operatorname{im}\left(p_{2}\right)=\left\{F \in \mathcal{W}_{1}^{\perp} \mid p_{2}(F)=F\right\}, \quad \mathcal{W}_{2}^{\perp}=\operatorname{ker}\left(p_{2}\right)=\left\{F \in \mathcal{W}_{1}^{\perp} \mid p_{2}(F)=0\right\} .
$$

Finally, we consider the operator $p_{3}: \mathcal{W}_{2}^{\perp} \rightarrow \mathcal{W}_{2}^{\perp}$ defined by

$$
p_{3}(F)(x, y, z)=\eta(x) F\left(\xi, \varphi^{2} y, \varphi^{2} z\right)
$$

and we get the following

Lemma 2.3. The operator $p_{3}$ has the properties:

(i) $p_{3} \circ p_{3}=p_{3}$; 
(ii) $\left\langle p_{3}\left(F^{\prime}\right), F^{\prime \prime}\right\rangle=\left\langle F^{\prime}, p_{3}\left(F^{\prime \prime}\right)\right\rangle, \quad F^{\prime}, F^{\prime \prime} \in \mathcal{W}_{2}^{\perp}$;

(iii) $p_{3} \circ(\lambda a)=(\lambda a) \circ p_{3}$.

By virtue of Lemma 2.3 we have

$$
\mathcal{W}_{3}=\operatorname{im}\left(p_{3}\right)=\left\{F \in \mathcal{W}_{2}^{\perp} \mid p_{3}(F)=F\right\}, \quad \mathcal{W}_{4}=\operatorname{ker}\left(p_{3}\right)=\left\{F \in \mathcal{W}_{2}^{\perp} \mid p_{3}(F)=0\right\} .
$$

From Lemma2.1, Lemma2.2 and Lemma 2.3 we have immediately

Theorem 2.4. The decomposition $\mathcal{F}=\mathcal{W}_{1} \oplus \mathcal{W}_{2} \oplus \mathcal{W}_{3} \oplus \mathcal{W}_{4}$ is orthogonal and invariant under the action of $\mathcal{G}$. The subspaces $\mathcal{W}_{i}(i=1,2,3,4)$ are determined by

$$
\begin{aligned}
& \mathcal{W}_{1}: F(x, y, z)=-F\left(\varphi^{2} x, \varphi^{2} y, \varphi^{2} z\right), \\
& \mathcal{W}_{2}: F(x, y, z)=\eta(y) F\left(\varphi^{2} x, \xi, \varphi^{2} z\right)+\eta(z) F\left(\varphi^{2} x, \varphi^{2} y, \xi\right), \\
& \mathcal{W}_{3}: F(x, y, z)=\eta(x) F\left(\xi, \varphi^{2} y, \varphi^{2} z\right), \\
& \mathcal{W}_{4}: F(x, y, z)=-\eta(x)\left\{\eta(y) F\left(\xi, \xi, \varphi^{2} z\right)+\eta(z) F\left(\xi, \varphi^{2} y, \xi\right)\right\} .
\end{aligned}
$$

Obviously, we have

$$
F=p_{1}(F)+p_{2}(F)+p_{3}(F)+p_{4}(F) .
$$

Corollary 2.5. The subspaces $\mathcal{W}_{i}(i=1,2,3,4)$ are characterized as follows:

$$
\begin{aligned}
& \mathcal{W}_{1}=\{F \in \mathcal{F} \mid F(v(x), y, z)=F(x, v(y), z)=F(x, y, v(z))=0\}, \\
& \mathcal{W}_{2}=\{F \in \mathcal{F} \mid F(v(x), y, z)=F(x, h(y), h(z))=0\}, \\
& \mathcal{W}_{3}=\{F \in \mathcal{F} \mid F(h(x), y, z)=F(x, v(y), z)=F(x, y, v(z))=0\}, \\
& \mathcal{W}_{4}=\{F \in \mathcal{F} \mid F(h(x), y, z)=F(x, h(y), h(z))=0\} .
\end{aligned}
$$

According to (13), (15) and (4) we obtain the following

Corollary 2.6. The Lee forms of $F$ have the following properties in each of the subspaces $\mathcal{W}_{i}(i=1,2,3,4)$ :

(i) If $F \in \mathcal{W}_{1}$, then $\theta \circ v=\theta^{*} \circ v=\omega=0$;

(ii) If $F \in \mathcal{W}_{2}$, then $\theta \circ h=\theta^{*} \circ h=\omega=0$;

(iii) If $F \in \mathcal{W}_{3}$, then $\theta=\theta^{*}=\omega=0$;

(iv) If $F \in \mathcal{W}_{4}$, then $\theta=\theta^{*}=0$.

Further we continue the decomposition of the subspaces $\mathcal{W}_{i}(i=1,2,3,4)$ of $\mathcal{F}$.

2.1. The decomposition of $\mathcal{W}_{1}$. Let us consider the $2 n$-dimensional distribution $H=\operatorname{ker}(\eta)$ of the tangent bundle of $(M, \varphi, \xi, \eta, g)$, the endomorphism $J=\left.\varphi\right|_{H}$ and the metric $h=\left.g\right|_{H}$, where $\left.\varphi\right|_{H}$ and $\left.g\right|_{H}$ are the restrictions of $\varphi$ and $g$ on $H$, respectively. Let us remark that $J$ and $h$ are an almost complex structure and a Norden metric on $H$, respectively, i.e.

$$
J^{2}=-\mathrm{Id}, \quad h(J x, J y)=-h(x, y) .
$$

Then $(H, J, h)$ can be considered as an almost complex manifold with Norden metric.

Moreover, the subspace $\mathcal{W}_{1}$ coincides with the restriction of $\mathcal{F}$ on $H$. By this reason the decomposition of $\mathcal{W}_{1}$ is made as the decomposition, known from [2], of the corresponding space of $\mathcal{F}$ for an almost complex manifold with Norden metric. Then we obtain the following 
Proposition 2.7. Let $F \in \mathcal{F}$ and $F_{i}(i=1,2,3)$ be the projections of $F$ on the subspaces $\mathcal{F}_{i}$, respectively. Then

$$
\begin{aligned}
F_{1}(x, y, z)= & \frac{1}{2 n}\left\{g(\varphi x, \varphi y) \theta\left(\varphi^{2} z\right)+g(x, \varphi y) \theta(\varphi z)+g(\varphi x, \varphi z) \theta\left(\varphi^{2} y\right)+g(x, \varphi z) \theta(\varphi y)\right\} ; \\
F_{2}(x, y, z)= & -\frac{1}{4}\left\{F\left(\varphi^{2} x, \varphi^{2} y, \varphi^{2} z\right)+F\left(\varphi^{2} y, \varphi^{2} z, \varphi^{2} x\right)-F\left(\varphi y, \varphi^{2} z, \varphi x\right)+F\left(\varphi^{2} x, \varphi^{2} z, \varphi^{2} y\right)\right. \\
& \left.+F\left(\varphi^{2} z, \varphi^{2} y, \varphi^{2} x\right)-F\left(\varphi z, \varphi^{2} y, \varphi x\right)\right\}-\frac{1}{2 n}\left\{g(\varphi x, \varphi y) \theta\left(\varphi^{2} z\right)+g(x, \varphi y) \theta(\varphi z)\right. \\
& \left.+g(\varphi x, \varphi z) \theta\left(\varphi^{2} y\right)+g(x, \varphi z) \theta(\varphi y)\right\} ; \\
F_{3}(x, y, z)= & -\frac{1}{4}\left\{F\left(\varphi^{2} x, \varphi^{2} y, \varphi^{2} z\right)-F\left(\varphi^{2} y, \varphi^{2} z, \varphi^{2} x\right)+F\left(\varphi y, \varphi^{2} z, \varphi x\right)+F\left(\varphi^{2} x, \varphi^{2} z, \varphi^{2} y\right)\right. \\
& \left.-F\left(\varphi^{2} z, \varphi^{2} y, \varphi^{2} x\right)+F\left(\varphi z, \varphi^{2} y, \varphi x\right)\right\} .
\end{aligned}
$$

Therefore, the component of $F$ on $W_{1}$ is

$$
p_{1}(F)=F_{1}+F_{2}+F_{3} \text {. }
$$

2.2. The decomposition of $\mathcal{W}_{2}$. Let us consider linear operators $L_{j}: \mathcal{W}_{2} \rightarrow \mathcal{W}_{2}(j=1,2)$ defined by

$$
\begin{aligned}
& L_{1}(F)(x, y, z)=F(\varphi x, \varphi y, \xi) \eta(z)+F(\varphi x, \varphi z, \xi) \eta(y), \\
& L_{2}(F)(x, y, z)=F\left(\varphi^{2} y, \varphi^{2} x, \xi\right) \eta(z)+F\left(\varphi^{2} z, \varphi^{2} x, \xi\right) \eta(y) .
\end{aligned}
$$

It is easy to check the following

Lemma 2.8. The linear operator $L_{j}(j=1,2)$ is an involutive isometry on $W_{2}$ and it is invariant with respect to the group $\mathcal{G}$, i.e.

$$
L_{j} \circ L_{j}=\operatorname{Id}_{W_{2}}, \quad\left\langle L_{j}\left(F^{\prime}\right), L_{j}\left(F^{\prime \prime}\right)\right\rangle=\left\langle F^{\prime}, F^{\prime \prime}\right\rangle, \quad L_{j}((\lambda a) F)=(\lambda a)\left(L_{j}(F)\right),
$$

where $F^{\prime}, F^{\prime \prime} \in \mathcal{W}_{2}$ and $\lambda a$ is determined by (7).

Therefore, $L_{1}$ has two eigenvalues +1 and -1 , and the corresponding eigenspaces

$$
\mathcal{W}_{2}^{+}=\left\{F \in \mathcal{W}_{2} \mid L_{1}(F)=F\right\}, \quad \mathcal{W}_{2}^{-}=\left\{F \in \mathcal{W}_{2} \mid L_{1}(F)=-F\right\}
$$

are invariant orthogonal subspaces of $\mathcal{W}_{2}$.

In order to decompose $\mathcal{W}_{2}^{+}$and $\mathcal{W}_{2}^{-}$, we use the operator $L_{2}$ on $\mathcal{W}_{2}^{+}$and $\mathcal{W}_{2}^{-}$, respectively. Let us denote the corresponding eigenspaces $\mathcal{W}_{2, k}(k=1,2,3,4)$ by

$$
\begin{array}{ll}
\mathcal{W}_{2,1}=\left\{\mathcal{W}_{2}^{+} \mid L_{2}(F)=-F\right\}, & \mathcal{W}_{2,2}=\left\{\mathcal{W}_{2}^{-} \mid L_{2}(F)=-F\right\}, \\
\mathcal{W}_{2,3}=\left\{\mathcal{W}_{2}^{+} \mid L_{2}(F)=F\right\}, & \mathcal{W}_{2,4}=\left\{\mathcal{W}_{2}^{-} \mid L_{2}(F)=F\right\} .
\end{array}
$$

Thus we establish the truthfulness of the following

Theorem 2.9. The decomposition $\boldsymbol{W}_{2}=\mathcal{W}_{2,1} \oplus \mathcal{W}_{2,2} \oplus \mathcal{W}_{2,3} \oplus \mathcal{W}_{2,4}$ is orthogonal and invariant with respect to the structure group.

Proposition 2.10. Let $F \in \mathcal{F}$ and $F_{j}(j=4, \ldots, 9)$ be the projections of $\mathcal{F}$ in the classes $\mathcal{F}_{j}$. Then we have

$$
\begin{aligned}
& F_{4}(x, y, z)=-\frac{\theta(\xi)}{2 n}\{g(\varphi x, \varphi y) \eta(z)+g(\varphi x, \varphi z) \eta(y)\} \\
& F_{5}(x, y, z)=-\frac{\theta^{*}(\xi)}{2 n}\{g(x, \varphi y) \eta(z)+g(x, \varphi z) \eta(y)\} ; \\
& F_{6}(x, y, z)=\frac{\theta(\xi)}{2 n}\{g(\varphi x, \varphi y) \eta(z)+g(\varphi x, \varphi z) \eta(y)\}+\frac{\theta^{*}(\xi)}{2 n}\{g(x, \varphi y) \eta(z)+g(x, \varphi z) \eta(y)\}
\end{aligned}
$$


(20b)

$$
\begin{aligned}
& +\frac{1}{4}\left[F\left(\varphi^{2} x, \varphi^{2} y, \xi\right)+F\left(\varphi^{2} y, \varphi^{2} x, \xi\right)-F(\varphi x, \varphi y, \xi)-F(\varphi y, \varphi x, \xi)\right] \eta(z) \\
& +\frac{1}{4}\left[F\left(\varphi^{2} x, \varphi^{2} z, \xi\right)+F\left(\varphi^{2} z, \varphi^{2} x, \xi\right)-F(\varphi x, \varphi z, \xi)-F(\varphi z, \varphi x, \xi)\right] \eta(y) ; \\
F_{7}(x, y, z)= & \frac{1}{4}\left[F\left(\varphi^{2} x, \varphi^{2} y, \xi\right)-F\left(\varphi^{2} y, \varphi^{2} x, \xi\right)-F(\varphi x, \varphi y, \xi)+F(\varphi y, \varphi x, \xi)\right] \eta(z) \\
& +\frac{1}{4}\left[F\left(\varphi^{2} x, \varphi^{2} z, \xi\right)-F\left(\varphi^{2} z, \varphi^{2} x, \xi\right)-F(\varphi x, \varphi z, \xi)+F(\varphi z, \varphi x, \xi)\right] \eta(y) ; \\
F_{8}(x, y, z)= & \frac{1}{4}\left[F\left(\varphi^{2} x, \varphi^{2} y, \xi\right)+F\left(\varphi^{2} y, \varphi^{2} x, \xi\right)+F(\varphi x, \varphi y, \xi)+F(\varphi y, \varphi x, \xi)\right] \eta(z) \\
& +\frac{1}{4}\left[F\left(\varphi^{2} x, \varphi^{2} z, \xi\right)+F\left(\varphi^{2} z, \varphi^{2} x, \xi\right)+F(\varphi x, \varphi z, \xi)+F(\varphi z, \varphi x, \xi)\right] \eta(y) ; \\
F_{9}(x, y, z)= & \frac{1}{4}\left[F\left(\varphi^{2} x, \varphi^{2} y, \xi\right)-F\left(\varphi^{2} y, \varphi^{2} x, \xi\right)+F(\varphi x, \varphi y, \xi)-F(\varphi y, \varphi x, \xi)\right] \eta(z) \\
& +\frac{1}{4}\left[F\left(\varphi^{2} x, \varphi^{2} z, \xi\right)-F\left(\varphi^{2} z, \varphi^{2} x, \xi\right)+F(\varphi x, \varphi z, \xi)-F(\varphi z, \varphi x, \xi)\right] \eta(y) .
\end{aligned}
$$

Proof. Lemma 2.8 implies that the tensor $\frac{1}{2}\left\{F+L_{1}(F)\right\}$ is the projection of $F \in \mathcal{W}_{2}$ in $\mathcal{W}_{2}^{+}=$ $\mathcal{W}_{2,1} \oplus \mathcal{W}_{2,3}$ and moreover $\frac{1}{2}\left\{F-L_{2}(F)\right\}$ is the projection of $F \in \mathcal{W}_{2}^{+}$in $\mathcal{W}_{2,1}$. Thus, we find the expression of the projection $p_{2,1}$ of $F$ from $\mathcal{W}_{2}$ to $\mathcal{W}_{2,1}$ in terms of $L_{1}$ and $L_{2}$, namely

$$
p_{2,1}(F)=\frac{1}{4}\left\{F-L_{1}(F)+L_{2}(F)-L_{2} \circ L_{1}(F)\right\} .
$$

In a similar way we treat with the projections $p_{2, k}(F)$ in $\mathcal{W}_{2, k}(k=2,3,4)$. After that, using (19), we get the following expressions

$$
\begin{aligned}
p_{2,1}(F)(x, y, z)= & \frac{1}{4}\left[F\left(\varphi^{2} x, \varphi^{2} y, \xi\right)+F\left(\varphi^{2} y, \varphi^{2} x, \xi\right)-F(\varphi x, \varphi y, \xi)-F(\varphi y, \varphi x, \xi)\right] \eta(z) \\
& +\frac{1}{4}\left[F\left(\varphi^{2} x, \varphi^{2} z, \xi\right)+F\left(\varphi^{2} z, \varphi^{2} x, \xi\right)-F(\varphi x, \varphi z, \xi)-F(\varphi z, \varphi x, \xi)\right] \eta(y) ; \\
p_{2,2}(F)(x, y, z)= & \frac{1}{4}\left[F\left(\varphi^{2} x, \varphi^{2} y, \xi\right)-F\left(\varphi^{2} y, \varphi^{2} x, \xi\right)-F(\varphi x, \varphi y, \xi)+F(\varphi y, \varphi x, \xi)\right] \eta(z) \\
& +\frac{1}{4}\left[F\left(\varphi^{2} x, \varphi^{2} z, \xi\right)-F\left(\varphi^{2} z, \varphi^{2} x, \xi\right)-F(\varphi x, \varphi z, \xi)+F(\varphi z, \varphi x, \xi)\right] \eta(y) ; \\
p_{2,3}(F)(x, y, z)= & \frac{1}{4}\left[F\left(\varphi^{2} x, \varphi^{2} y, \xi\right)+F\left(\varphi^{2} y, \varphi^{2} x, \xi\right)+F(\varphi x, \varphi y, \xi)+F(\varphi y, \varphi x, \xi)\right] \eta(z) \\
& +\frac{1}{4}\left[F\left(\varphi^{2} x, \varphi^{2} z, \xi\right)+F\left(\varphi^{2} z, \varphi^{2} x, \xi\right)+F(\varphi x, \varphi z, \xi)+F(\varphi z, \varphi x, \xi)\right] \eta(y) ; \\
p_{2,4}(F)(x, y, z)= & \frac{1}{4}\left[F\left(\varphi^{2} x, \varphi^{2} y, \xi\right)-F\left(\varphi^{2} y, \varphi^{2} x, \xi\right)+F(\varphi x, \varphi y, \xi)-F(\varphi y, \varphi x, \xi)\right] \eta(z) \\
& +\frac{1}{4}\left[F\left(\varphi^{2} x, \varphi^{2} z, \xi\right)-F\left(\varphi^{2} z, \varphi^{2} x, \xi\right)+F(\varphi x, \varphi z, \xi)-F(\varphi z, \varphi x, \xi)\right] \eta(y) .
\end{aligned}
$$

By virtue of Corollary 2.6. (4) and (21), we establish that the Lee forms $\theta$ and $\theta^{*}$ of $F$ are zero in $\mathcal{W}_{2, k}(k=2,3,4)$. We have no additional conditions for $\theta$ and $\theta^{*}$ in $\mathcal{W}_{2,1}$. Then, $\mathcal{W}_{2,1}$ can be additionally decomposed to three subspaces determined by the conditions $\theta=0, \theta^{*}=0$ and $\theta=\theta^{*}=0$, respectively, i.e.

$$
\mathcal{W}_{2,1}=\mathcal{W}_{2,1,1} \oplus \mathcal{W}_{2,1,2} \oplus \mathcal{W}_{2,1,3}
$$


where

$$
\begin{gathered}
\mathcal{W}_{2,1,1}=\left\{F \in \mathcal{W}_{2,1} \mid \theta^{*}=0\right\}, \quad \mathcal{W}_{2,1,2}=\left\{F \in \mathcal{W}_{2,1} \mid \theta=0\right\}, \\
\mathcal{W}_{2,1,3}=\left\{F \in \mathcal{W}_{2,1} \mid \theta=0, \theta^{*}=0\right\} .
\end{gathered}
$$

According to (5), the classes $\mathcal{F}_{4}$ and $\mathcal{F}_{5}$ are defined by explicit expressions of $F$, which have the form of $p_{2,1}(F)$ in (21). Hence we conclude that $\mathcal{F}_{4}$ and $\mathcal{F}_{5}$ are the subspaces $\mathcal{W}_{2,1,1}$ and $\mathcal{W}_{2,1,2}$ of $W_{2,1}$, respectively, and the projections of $F$ on them have the form given in the first line of (20). Therefore, the equality for $F_{6}$ in (20) follows from the form of $p_{2,1}(F)$ in (21) and the fact that $\mathcal{F}_{6}$ coincides with $\mathcal{W}_{2,1,3}$.

The form of $p_{2,2}(F), p_{2,3}(F)$ and $p_{2,4}(F)$ in (21) satisfies the conditions in (5) for the subspace $\mathcal{F}_{7}$, $\mathcal{F}_{8}$ and $\mathcal{F}_{9}$, respectively.

Thus, the subspaces $\mathcal{W}_{2,1,1}, \mathcal{W}_{2,1,2}, \mathcal{W}_{2,1,3}, \mathcal{W}_{2,2}, \mathcal{W}_{2,3}, \mathcal{W}_{2,4}$ correspond to the classes $\mathcal{F}_{4}, \mathcal{F}_{5}$, $\mathcal{F}_{6}, \mathcal{F}_{7}, \mathcal{F}_{8}, \mathcal{F}_{9}$, respectively.

Therefore, the component of $F$ on $\mathcal{W}_{2}$ is

$$
p_{2}(F)=F_{4}+F_{5}+F_{6}+F_{7}+F_{8}+F_{9} .
$$

2.3. The decomposition of $\mathcal{W}_{3}$ and $\mathcal{W}_{4}$. Finally, since $\mathcal{F}_{10}$ and $\mathcal{F}_{11}$ are determined in (5) by an expression of $F$ which coincide with the conditions in (13) for $\mathcal{W}_{3}$ and $\mathcal{W}_{4}$, respectively, we have the following

Proposition 2.11. Let $F \in \mathcal{F}$ and $F_{l}(l=10,11)$ be the projections of $\mathcal{F}$ in the subspaces $\mathcal{F}_{l}$. Then we have

$$
\begin{aligned}
& F_{10}(x, y, z)=\eta(x) F\left(\xi, \varphi^{2} y, \varphi^{2} z\right) ; \\
& F_{11}(x, y, z)=-\eta(x)\left\{\eta(y) F\left(\xi, \xi, \varphi^{2} z\right)+\eta(z) F\left(\xi, \varphi^{2} y, \xi\right)\right\} .
\end{aligned}
$$

Therefore, the components of $F$ on $\mathcal{W}_{3}$ and $\mathcal{W}_{4}$ are

$$
p_{3}(F)=F_{10}, \quad p_{4}(F)=F_{11},
$$

respectively. Then, bearing in mind (18), (22) and (24), we obtain that

$$
F(x, y, z)=\sum_{i=1}^{11} F_{i}(x, y, z)
$$

where the components $F_{i}$ of $F$ in the corresponding subspaces $\mathcal{F}_{i}(i=1, \ldots, 11)$ of $\mathcal{F}$ are determined in Proposition 2.7, Proposition 2.10 and Proposition 2.11.

In conclusion we give

Theorem 2.12. The almost contact B-metric manifold $(M, \varphi, \xi, \eta, g)$ belongs to the basic class $\mathcal{F}_{i}(i=$ $1, \ldots, 11)$ if and only if the structure tensor $F$ satisfies the condition $F=F_{i}$, where the components $F_{i}$ of $F$ are given in (17), (20) and (23).

It is easy to conclude that an almost contact B-metric manifold belongs to a direct sum of two or more basic classes, i.e. $(M, \varphi, \xi, \eta, g) \in \mathcal{F}_{i} \oplus \mathcal{F}_{j} \oplus \cdots$, if and only if the structure tensor $F$ on $(M, \varphi, \xi, \eta, g)$ is the sum of the corresponding components $F_{i}, F_{j}, \ldots$ of $F$, i.e. the following condition is satisfied $F=F_{i}+F_{j}+\cdots$.

\section{THE COMPONENTS OF THE STRUCTURE TENSOR FOR DIMENSION 3}

In this section we are interesting in the lowest dimension of the manifolds under consideration, i.e. we consider the case of $\operatorname{dim} M=3$ for $(M, \varphi, \xi, \eta, g)$.

Let us denote the components $F_{i j k}=F\left(e_{i}, e_{j}, e_{k}\right)$ of the structure tensor $F$ with respect to a $\varphi$-basis $\left\{e_{i}\right\}_{i=0}^{2}=\left\{e_{0}=\xi, e_{1}=e, e_{2}=\varphi e\right\}$, which satisfies the following conditions

$$
g\left(e_{0}, e_{0}\right)=g\left(e_{1}, e_{1}\right)=-g\left(e_{2}, e_{2}\right)=1, \quad g\left(e_{i}, e_{j}\right)=0, i \neq j \in\{0,1,2\} .
$$


Then, using (4) and (25), we obtain the components of the Lee forms with respect to the basis $\left\{e_{i}\right\}_{i=0}^{2}$ as follows

$$
\begin{array}{lll}
\theta_{0}=F_{110}-F_{220}, & \theta_{1}=F_{111}-F_{221}, & \theta_{2}=F_{112}-F_{211}, \\
\theta_{0}^{*}=F_{120}+F_{210}, & \theta_{1}^{*}=F_{112}+F_{211}, & \theta_{2}^{*}=F_{111}+F_{221}, \\
\omega_{0}=0, & \omega_{1}=F_{001}, & \omega_{2}=F_{002} .
\end{array}
$$

Let us consider arbitrary vectors $x, y, z \in T_{p} M$. Therefore we have $x=x^{i} e_{i}, y=y^{i} e_{i}, z=z^{i} e_{i}$ with respect to $\left\{e_{i}\right\}_{i=0}^{2}$.

By direct computations we obtain

Proposition 3.1. The components $F_{i}(i=1,2, \ldots, 11)$ of the structure tensor $F$ in the corresponding basic classes $\mathcal{F}_{i}$ are the following

$$
\begin{aligned}
& F_{1}(x, y, z)=\left(x^{1} \theta_{1}-x^{2} \theta_{2}\right)\left(y^{1} z^{1}+y^{2} z^{2}\right), \quad \theta_{1}=F_{111}=F_{122}, \quad \theta_{2}=-F_{211}=-F_{222} \\
& F_{2}(x, y, z)=F_{3}(x, y, z)=0 ; \\
& F_{4}(x, y, z)=\frac{1}{2} \theta_{0}\left\{x^{1}\left(y^{0} z^{1}+y^{1} z^{0}\right)-x^{2}\left(y^{0} z^{2}+y^{2} z^{0}\right)\right\}, \quad \frac{1}{2} \theta_{0}=F_{101}=F_{110}=-F_{202}=-F_{220} \\
& F_{5}(x, y, z)=\frac{1}{2} \theta_{0}^{*}\left\{x^{1}\left(y^{0} z^{2}+y^{2} z^{0}\right)+x^{2}\left(y^{0} z^{1}+y^{1} z^{0}\right)\right\}, \quad \frac{1}{2} \theta_{0}^{*}=F_{102}=F_{120}=F_{201}=F_{210} \\
& F_{6}(x, y, z)=F_{7}(x, y, z)=0 ; \\
& F_{8}(x, y, z)=\lambda\left\{x^{1}\left(y^{0} z^{1}+y^{1} z^{0}\right)+x^{2}\left(y^{0} z^{2}+y^{2} z^{0}\right)\right\}, \quad \lambda=F_{101}=F_{110}=F_{202}=F_{220} ; \\
& F_{9}(x, y, z)=\mu\left\{x^{1}\left(y^{0} z^{2}+y^{2} z^{0}\right)-x^{2}\left(y^{0} z^{1}+y^{1} z^{0}\right)\right\}, \quad \mu=F_{102}=F_{120}=-F_{201}=-F_{210} ; \\
& F_{10}(x, y, z)=v x^{0}\left(y^{1} z^{1}+y^{2} z^{2}\right), \quad v=F_{011}=F_{022} ; \\
& F_{11}(x, y, z)=x^{0}\left\{\left(y^{1} z^{0}+y^{0} z^{1}\right) \omega_{1}+\left(y^{2} z^{0}+y^{0} z^{2}\right) \omega_{2}\right\}, \quad \omega_{1}=F_{010}=F_{001}, \quad \omega_{2}=F_{020}=F_{002} .
\end{aligned}
$$

Proof. Using Theorem 2.12 and the expressions of the components $F_{i}$ of $F$ for the corresponding classes $\mathcal{F}_{i}(i=1, \ldots, 11)$, determined by (17), (20) and (23), the equalities (25), (26) and the properties (3) of $F$, we obtain the corresponding explicit expression of $F_{i}$ for dimension 3.

According to Theorem 2.12 and Proposition 3.1, we obtain

Theorem 3.2. The class of almost contact B-metric manifolds of dimension 3 is

$$
\mathcal{F}_{1} \oplus \mathcal{F}_{4} \oplus \mathcal{F}_{5} \oplus \mathcal{F}_{8} \oplus \mathcal{F}_{9} \oplus \mathcal{F}_{10} \oplus \mathcal{F}_{11}
$$

i.e. the basic classes $\mathcal{F}_{2}, \mathcal{F}_{3}, \mathcal{F}_{6}, \mathcal{F}_{7}$ are restricted to the special class $\mathcal{F}_{0}$.

\section{Some eXamples}

4.1. Time-like sphere as a manifold from the class $\mathcal{F}_{4} \oplus \mathcal{F}_{5}$. In [3], an example of an almost contact manifold with B-metric is given. It is constructed as a time-like sphere of $\mathbb{R}^{2 n+2}$ with complex structure and Norden metric. Namely, let $\mathbb{R}^{2 n+2}=\left\{\left(u^{1}, \ldots, u^{n+1} ; v^{1}, \ldots, v^{n+1}\right) \mid u^{i}, v^{i} \in \mathbb{R}\right\}$ be considered as a complex Riemannian manifold with the canonical complex structure $J$ and the metric $g$ defined by

$$
J \frac{\partial}{\partial u^{i}}=\frac{\partial}{\partial v^{i}}, \quad J \frac{\partial}{\partial v^{i}}=-\frac{\partial}{\partial u^{i}}, \quad g(x, x)=-\delta_{i j} \lambda^{i} \lambda^{j}+\delta_{i j} \mu^{i} \mu^{j},
$$

where $x=\lambda \frac{\partial}{\partial u^{i}}+\mu \frac{\partial}{\partial v^{i}}$. Identifying the point $p$ in $\mathbb{R}^{2 n+2}$ with its position vector $Z$, we define the unit time-like sphere

$$
S^{2 n+1}: g(Z, Z)=-1 \text {. }
$$

The almost contact structure is determined by

$$
\xi=\sin t \cdot Z+\cos t \cdot J Z, \quad J x=\varphi x+\eta(x) J \xi,
$$


where $t=\arctan (g(z, J z)) \in\left(-\frac{\pi}{2} ; \frac{\pi}{2}\right)$ and $x, \varphi x \in T_{p} S^{2 n+1}$. The metric on the hypersurface is the restriction of $g$ and it is denote by the same letter. Then $\left(S^{2 n+1}, \varphi, \xi, \eta, g\right)$ is an almost contact B-metric manifold. It belongs to the class $\mathcal{F}_{4} \oplus \mathcal{F}_{5}$ because the structure tensor has the following form

$$
F(x, y, z)=-\cos t\{g(\varphi x, \varphi y) \eta(z)+g(\varphi x, \varphi z) \eta(y)\}-\sin t\{g(x, \varphi y) \eta(z)+g(x, \varphi z) \eta(y)\},
$$

where $\cos t=\frac{\theta(\xi)}{2 n}, \sin t=\frac{\theta^{*}(\xi)}{2 n}$ and then we obtain the following expression of $F$, bearing in mind (20):

$$
F=F_{4}+F_{5} .
$$

If we consider the 3-dimensional unit time-like sphere $\left(S^{3}, \varphi, \xi, \eta, g\right)$ then we have the following form of the structure tensor, using (27):

$$
F(x, y, z)=\frac{1}{2}\left\{\left(\theta_{0} x^{1}+\theta_{0}^{*} x^{2}\right)\left(y^{0} z^{1}+y^{1} z^{0}\right)+\left(\theta_{0}^{*} x^{1}-\theta_{0} x^{2}\right)\left(y^{0} z^{2}+y^{2} z^{0}\right)\right\}
$$

where $\frac{1}{2} \theta_{0}=F_{101}=F_{110}=-F_{202}=-F_{220}$ and $\frac{1}{2} \theta_{0}^{*}=F_{102}=F_{120}=F_{201}=F_{210}$ with respect to the orthonormal $\varphi$-basis $\left\{e_{i}\right\}_{i=0}^{2}$.

4.2. Lie group as a manifold from the class $\mathcal{F}_{9} \oplus \mathcal{F}_{10}$. Let $L$ be a $(2 n+1)$-dimensional real connected Lie group and its associated Lie algebra with a global basis $\left\{E_{0}, E_{1}, \ldots, E_{2 n}\right\}$ of left invariant vector fields on $L$ defined by

$$
\left[E_{0}, E_{i}\right]=-a_{i} E_{i}-a_{n+i} E_{n+i}, \quad\left[E_{0}, E_{n+i}\right]=-a_{n+i} E_{i}+a_{i} E_{n+i},
$$

where $a_{1}, \ldots, a_{2 n}$ are real constants and $\left[E_{j}, E_{k}\right]=0$ in other cases. Let an invariant almost contact structure be defined for $i \in\{1, \ldots, n\}$ by

$$
\varphi E_{0}=0, \quad \varphi E_{i}=E_{n+i}, \quad \varphi E_{n+i}=-E_{i}, \quad \xi=E_{0}, \quad \eta\left(E_{0}\right)=1, \quad \eta\left(E_{i}\right)=\eta\left(E_{n+i}\right)=0 .
$$

Let $g$ be a pseudo-Riemannian metric such that for $i \in\{1, \ldots, n\}$ and $j, k \in\{1, \ldots, 2 n\}, j \neq k$ the following equalities are valid

$$
g\left(E_{0}, E_{0}\right)=g\left(E_{i}, E_{i}\right)=-g\left(E_{n+i}, E_{n+i}\right)=1, \quad g\left(E_{0}, E_{j}\right)=g\left(E_{j}, E_{k}\right)=0 .
$$

Thus, because of (1), the induced $(2 n+1)$-dimensional manifold $(L, \varphi, \xi, \eta, g)$ is an almost contact B-metric manifold.

Let us remark that in [9] the same Lie group with the same almost contact structure but equipped with a compatible Riemannian metric is studied as an almost cosymplectic manifold.

Let us consider the constructed almost contact B-metric manifold $(L, \varphi, \xi, \eta, g)$ in dimension 3 , i.e. for $n=1$.

By virtue of (28) and (30) for $n=1$, and using the Koszul equality

$$
2 g\left(\nabla_{E_{i}} E_{j}, E_{k}\right)=g\left(\left[E_{i}, E_{j}\right], E_{k}\right)+g\left(\left[E_{k}, E_{i}\right], E_{j}\right)+g\left(\left[E_{k}, E_{j}\right], E_{i}\right)
$$

for the Levi-Civita connection $\nabla$ of $g$, we obtain

(32) $\nabla_{E_{1}} E_{1}=\nabla_{E_{2}} E_{2}=-a_{1} E_{0}, \quad \nabla_{E_{0}} E_{1}=-a_{2} E_{2}, \quad \nabla_{E_{0}} E_{2}=-a_{2} E_{1}, \quad \nabla_{E_{1}} E_{0}=a_{1} E_{1}, \quad \nabla_{E_{2}} E_{0}=-a_{1} E_{2}$.

Then, using the latter equalities, (29) and (2), we get the following nonzero components $F_{i j k}=$ $F\left(E_{i}, E_{j}, E_{k}\right)$ of the structure tensor:

$$
F_{011}=F_{022}=-2 a_{2}, \quad F_{102}=F_{120}=-F_{201}=-F_{210}=a_{1} .
$$

Thus, we establish the following form of $F$ for arbitrary vectors $x=x^{i} E_{i}, y=y^{i} E_{i}, z=z^{i} E_{i}$

$$
F(x, y, z)=-2 a_{2} x^{0}\left(y^{1} z^{1}+y^{2} z^{2}\right)+a_{1}\left\{z^{0}\left(x^{1} y^{2}-x^{2} y^{1}\right)+y^{0}\left(x^{1} z^{2}-x^{2} z^{1}\right)\right\} .
$$

The latter equality implies that $F$ is represented in the form

$$
F(x, y, z)=F_{9}(x, y, z)+F_{10}(x, y, z),
$$


bearing in mind (27) for $\mu=a_{1}, v=-2 a_{2}$; or alternatively, the corresponding equalities from (20) and (23). Therefore, we prove that the constructed 3-dimensional manifold belongs to the class $\mathcal{F}_{9} \oplus \mathcal{F}_{10}$.

\section{References}

[1] V. Alexiev, G. Ganchev, On the classification of almost contact metric manifolds, In: Math. and Educ in Math., Proc. of 15th Spring Conf. of UBM, Sunny Beach, 1986, 155-161.

[2] G. Ganchev, A. Borisov, Note on the almost complex manifolds with a Norden metric, C. R. Acad. Bulgare Sci. 39 (1986) 31-34.

[3] G. Ganchev, V. Mihova, K. Gribachev, Almost contact manifolds with B-metric, Math. Balk. 7 (3-4) (1993) $261-276$.

[4] A. Gray, L. Hervella, The sixteen classes of almost Hermitian manifolds and their linear invariants, Ann. Mat. Pura Appl. 123 (1980) 35-58.

[5] M. Manev, Almost contact B-metric hypersurfaces of Kaehlerian manifolds with B-metric. In: Perspectives of Complex analysis, Differential Geometry and Mathematical Physics, Eds. St. Dimiev and K. Sekigawa, World Sci. Publ., Singapore, 2001, 159-170.

[6] M. Manev, M. Staikova, On almost paracontact Riemannian manifolds of type (n,n), J. Geom. 72 (2001) $108-114$.

[7] G. Nakova, S. Zamkovoy, Eleven classes of almost paracontact manifolds with semi-Riemannian metric of $(n+1, n)$, In: Adachi T., Hashimoto H., Hristov M. (Eds.), Recent Progress in Differential Geometry and its Related Fields, World Scientific Publ., Singapore, 2012, 119-136.

[8] A. M. Naveira, A classification of Riemannian almost product structures, Rend. Mat. Roma 3 (1983) 577-592.

[9] Z. Olszak, On almost cosymplectic manifolds, Kodai Math. J. 4 (2) (1981) 239-250.

[10] M.Staikova, K. Gribachev, Canonical connections and their conformal invariants on Riemannian P-manifolds, Serdica Math. J. 18 (1992) 150-161.

Medical University of Plovdiv, Faculty of Pharmacy, Department of Pharmaceutical Sciences, 15-A Vasil Aprilov BLVD.

E-mail address: hmanev@uni-plovdiv.bg 